\title{
Interaction of CREB and PGC-1 $\alpha$ Induces Fibronectin Type III Domain-Containing Protein 5 Expression in C2C12 Myotubes
}

\author{
Xiu-ying Yanga,b Margaret C.L. Tse ${ }^{c} \quad$ Xiang Hu$^{\mathrm{b}} \quad$ Wei-hua Jia ${ }^{\mathrm{a}}$ \\ Guan-hua Du ${ }^{a}$ Chi Bun Chan ${ }^{d}$ \\ aState Key Laboratory of Bioactive Substance and Function of Natural Medicines and Beijing Key \\ Laboratory of Drug Target and Screening Research, Institute of Materia Medica of Peking Union \\ Medical College, Beijing, China, 'bepartment of Physiology, University of Oklahoma Health Sciences \\ Center, Oklahoma City, USA, 'School of Biomedical Sciences, Li Ka Shing Faculty of Medicine, The \\ University of Hong Kong, Hong Kong, 'SChool of Biological Sciences, the University of Hong Kong, \\ 5N10 Kadoorie Biological Sciences Building, Hong Kong, China
}

\section{Key Words}

FNDC5 • Irisin $\cdot$ PGC-1 $\alpha$ Myokine $\cdot$ C2C12 $\cdot$ Skeletal muscle $\cdot$ Glucose

\begin{abstract}
Background/Aims: Fibronectin type III domain-containing protein 5 (FNDC5), also known as irisin, is a myokine secreted from muscle in response to exercise. However, the molecular mechanisms that regulate FNDC5 expression and the functional significance of irisn in skeletal muscle remain unknown. In this study, we explored the potential pathways that induce FNDC5 expression and delineated the metabolic effects of irisin on skeletal muscle. Methods: $\mathrm{C} 2 \mathrm{C} 12$ myotubes were treated with drugs at various concentrations and durations. The expression and activation of genes were measured by real-time polymerase chain reaction (qRT-PCR) and Western blotting. Oxidative phosphorylation was quantified by measuring the oxygen consumption rate (OCR). Results: We found that the exercise-mimicking treatment (CAMP, forskolin and isoproterenol) increased Fndc5 expression in C2C12 myotubes. CREB overexpressed $\mathrm{C2C} 12$ myotubes displayed higher Fndc5 expression. CREB over-expression also promoted peroxisome proliferator-activated receptor gamma coactivator $1 \alpha$ (PGC-1 $\alpha)$ expression. PGC-1 $\alpha$-induced $F n d c 5$ expression was blocked when the dominant negative form of CREB (S133A) was present. PGC-1 $\alpha$ mutation (S570A) also decreased Fndc5 expression. Immunoprecipitation showed that overexpressed PGC-1 $\alpha$ complexed with CREB in HEK293 cells. C2C12 myotubes treated with forskolin also increased endogenous CREB and PGC-1 $\alpha$ binding. Functionally, irisin treatment increased mitochondrial respiration, enhanced ATP production, promoted fatty acid oxidation but decreased glycolysis in myotubes. Conclusion: Our observation indicates that CAMP-mediated PGC-1 $\alpha /$ CREB interaction triggers Fndc5
\end{abstract}

Guan-hua Du

and Chi Bun Chan 


\section{Cellular Physiology Cell Physiol Biochem 2018;50:1574-1584

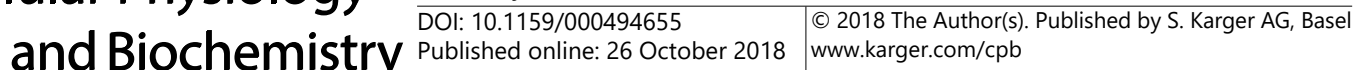

Yang et al.: CREB and PGC- $1 \alpha$ Control FNDC5 Expression

expression, which acts as an autocrine/paracrine to shape the metabolic phenotype of myotubes.

\section{Introduction}

Irisin, the secreted isoform of fibronectin type III domain-containing protein 5 (FNDC5), is a myokine that highly expresses in skeletal muscle, with relatively low expression in adipose tissue, pancreas, liver, and brain [1]. In humans, irisin is described as a metabolic myokine that improves obesity and glucose homeostasis by stimulating the browning of white adipose tissue (WAT) [2,3]. It also improves the endothelial functions in obese subjects [4]. In human skeletal muscle, treatment with irisin (50 nM) for $1 \mathrm{~h}$ increased glucose and fatty acid uptake, which was similar to insulin [5]. At lower concentration (5 $\mathrm{nM}$ ), irisin also stimulated mitochondrial biogenesis and increased UCP3 and GLUT4 levels in $\mathrm{C} 2 \mathrm{C} 12$ cells [6]. Moreover, irisin is an AMPK stimulator to promote $\beta$-oxidation in muscle [3]. Intraperitoneal injection of irisin ( $0.5 \mu \mathrm{g}$ per g of body weight) into obese and diabetic mice increased glucose uptake via stimulating GLUT4 translocation to the skeletal muscle cell membranes [7], suggesting irisin works as an autocrine/paracrine to modulate the energy homeostasis of skeletal muscle.

It was reported that peroxisome proliferator-activated receptor- $\gamma$ co-activator $1 \alpha$ (PGC$1 \alpha$ ) was a major regulator of FNDC5 expression because irisin was first isolated from the muscle of PGC- $1 \alpha$ transgenic mice $[2,8]$. PGC- $1 \alpha$ is an important regulator of mitochondrial biogenesis, which mediates various metabolic functions in response to different metabolic stresses [9]. Nevertheless, contradictory results were obtained from different laboratories that activation of PGC-1 $\alpha$ and FNDC5 expressions were uncoupled in skeletal muscle during exercise [10]. In 2017, a meta-analysis including 51 studies reported that a solid conclusion could not be made about the link between PGC-1 $\alpha$ activity and FNDC5 expression in response to physical activity [11]. Thus, the molecular mechanisms of exercise-regulated FNDC5 expression in skeletal muscle are still far from clear.

The present study aims to delineate the regulatory mechanism that controls FNDC5 expression as well as its metabolic functions in glucose and fatty acid metabolism in skeletal muscle.

\section{Materials and Methods}

\section{Chemicals and reagents}

C2C12 cells were purchased from ATCC (USA). Antibodies against pCREB S133 (cat. no. \#9198, diluted 1: 1500), CREB (cat. no. \#4820, diluted 1:1000), were purchased from Cell Signaling (USA). Anti-PGC-1 $\alpha$ (cat. no. \#8934, diluted 1:1000) was obtained from Abcam (USA). Anti-tubulin antibody (cat. no. \#T6074, diluted 1:1000) and anti-Flag antibody (cat. no. F3165, diluted 1:2000) were obtained from Sigma-Aldrich (USA). Anti-GFP antibody (cat. no. sc9996, diluted 1: 1000) was obtained from Santa Cruz Biotechnology (USA). Plasmids expressing wild-type CREB, the dominant-negative mutant of CREB (CREB S133A), wildtype PGC-1 $\alpha$, and the dominant-negative mutant of PGC- $1 \alpha$ (PGC-1 $\alpha$ 570A) were obtained from Addgene (USA). Other chemicals were purchased from Sigma-Aldrich (USA).

C2C12 cell culture and differentiation

C2C12 myoblasts were maintained in high-glucose DMEM with 5\% FBS, 15\% calf serum, $100 \mathrm{IU} / \mathrm{mL}$ of penicillin and $100 \mu \mathrm{g} / \mathrm{mL}$ of streptomycin (Invitrogen, USA). The culture protocol was strictly enforced to avoid cell confluence. Differentiation of myoblasts into myotubes was performed by incubating the confluent myoblasts with differentiating medium ( $2 \%$ horse serum, $100 \mathrm{I} . \mathrm{U} . / \mathrm{mL}$ penicillin, and $100 \mu \mathrm{g} / \mathrm{mL}$ streptomycin) for 4 days. Successful differentiation of the C2C12 was confirmed by morphological changes as previously reported [12].

\section{KARGER}




\section{Cellular Physiology Cell Physiol Biochem 2018;50:1574-1584 \begin{tabular}{ll|l} 
and Biochemistry & $\begin{array}{l}\text { DOI: 10.1159/000494655 } \\
\text { Published online: 26 October 2018 }\end{array}$ & $\begin{array}{l}\text { (c) } 2018 \text { The Author(s). Published by S. Karger AG, Basel } \\
\text { www.karger.com/cpb }\end{array}$ \\
\hline
\end{tabular}}

Yang et al.: CREB and PGC- $1 \alpha$ Control FNDC5 Expression

\section{Cell transfection}

C2C12 myotubes transfection was performed using Viromer RED as unstructured (Lipocalyx $\mathrm{GmbH}$, Germany). Briefly, transfection was done in cells seeded in a 6-well plate (80\% confluence) with complete antibiotic-free growth media. DNA (18 ng/ $\mu \mathrm{l})$ in $340 \mu \mathrm{l}$ solution was added to $60 \mu \mathrm{l}$ of viromer working solution. After mixing and incubation for approximately $15 \mathrm{~min}$ at room temperature, the solution was added to the cells. After 24-48 h, the medium was changed to $2 \%$ HS media to induce differentiation. Four days after transfection, the myotubes were collected for experiments.
Table 1. Primers used in real-time PCR

\begin{tabular}{lr}
\hline Genes & Primer sequence \\
\hline Mouse Fndc5 5' & ATGAAGGAGATGGGGAGGAA \\
Mouse Fndc5 3' & GCGGCAGAAGAGAGCTATAACA \\
Mouse Pgc-1 $\alpha 5^{\prime}$ & ATACCGCAAAGAGCACGAGAA \\
Mouse Pgc-1 $\alpha 3^{\prime}$ & CTCAAGAGCAGCGAAAGCGTCACA \\
Mouse Tfam 5' & CAGGAGGCAAAGGATGATTC \\
Mouse Tfam 3' & ATGTCTCCGGATCGTTTCAC \\
Mouse Nrf1 5' & TCTCACCCTCCAAACCCAAC \\
Mouse Nrf1 3' & ATGCTCTTGTACTTTCGCACCA \\
Mouse $\beta$-actin 5' & AACCGTGAAAAGATGACCCAGAT \\
Mouse $\beta$-actin 3' & CACAGCCTGGATGGCTACGT \\
\hline
\end{tabular}

Quantitative Real-time PCR

Total RNA was isolated using TRIzol Isolation Reagent (Invitrogen, USA). First-strand cDNA was synthesized using $1 \mu \mathrm{g}$ of total RNA and a reverse transcription reaction mix containing Superscript III reverse transcriptase (Invitrogen, USA) and Oligo-dT17 primer. The expression of genes was detected using RealMasterMix SYBR ROX (5 Prime Inc, USA) and the ABI7500 Real-time PCR System (Applied Biosystems, USA) with gene-specific primers pairs (Table 1). The results were quantified after normalization with $\beta$-actin [13].

\section{Mitochondrial respiration}

Mitochondrial respiration of C2C12 myotubes was determined by the Seahorse XFe 96 Extracellular Flux Analyzer using the XF Mito Stress Test Kit as previously described (Agilent, USA). The concentrations of oligomycin, carbonyl cyanide-p-trifluoromethoxyphenyl-hydrazone (FCCP), antimycin A and rotenone used were $100 \mu \mathrm{M}, 100 \mu \mathrm{M}, 100 \mu \mathrm{M}$ and $50 \mu \mathrm{M}$, respectively. The oxygen consumption rate (OCR) and extracellular acidification rate (ECAR) were recorded, and cellular respiration and ATP production were calculated as described by the manufacturer.

\section{Western blotting}

Tissue extracts were prepared by homogenizing the tissues in lysis buffer (50 mM Tris at pH 7.4, 40 $\mathrm{mM} \mathrm{NaCl}, 1 \mathrm{mM}$ EDTA, 0.5\% Triton X-100, $1.5 \mathrm{mM} \mathrm{Na}_{3} \mathrm{VO}_{4}, 50 \mathrm{mM} \mathrm{NaF}, 10 \mathrm{mM} \mathrm{Na} \mathrm{P}_{2} \mathrm{O}_{7}, 10 \mathrm{mM}$ sodium $\beta$-glycerol phosphate and protease inhibitor cocktail). Cell debris was removed by centrifugation, and the supernatants were collected for further analysis. Immunoblotting signals within the linear detection range were detected using the G:Box Chemi XRG imager (Syngene, USA) and were analysed by ImageJ (NIH, USA).

\section{Immunoprecipitation}

Lysis buffer washed Protein A/G-Agarose beads (50\% bead slurry; sc-2003; Santa Cruz, USA) were added to $500 \mu \mathrm{g}$ of cell lysate in a final volume of $500 \mu \mathrm{L}$. After incubation at $4^{\circ} \mathrm{C}$ for $60 \mathrm{~min}$, the lysates were centrifuged at $12,000 \mathrm{~g}$ for $1 \mathrm{~min}$ at $4^{\circ} \mathrm{C}$. The supernatant was transferred to a fresh tube with $2 \mu \mathrm{L}$ of primary antibody and $20 \mu \mathrm{L}$ protein $\mathrm{A} / \mathrm{G}$, followed by an overnight incubation at $4^{\circ} \mathrm{C}$ with gentle rocking. The Protein $A / G$ beads were then collected by centrifugation and washed three times with cell lysis buffer. After suspended in $20 \mu \mathrm{l}$ of $2 \times \operatorname{SDS}$ loading buffer and heated at $95^{\circ} \mathrm{C}$ for $5 \mathrm{~min}$, the supernatants were used for SDS-PAGE.

\section{Statistical analysis}

The results were expressed as means \pm S.E.M. and were considered significant when $P \leqslant 0.05$. Statistical analysis was performed using either Student's t-test or one-way ANOVA followed by Tukey's multiple comparison by the computer program Prism (GraphPad Software, USA). 


\section{Results}

\section{Exercise-mimic increased Fndc5 and Pgc-1 $\alpha$ expression in myotubes}

To reveal the mechanism of exercise-induced FNDC5 expression in skeletal muscle, we used Sp-cAMP [14, 15], forskolin [15], and isoproterenol [16, 17] stimulation to mimic the pathways that are induced by exercise. Sp-cAMP, a cell-permeable analogue of adenosine 3',5'-cylcic monophosphorothioate (cAMP), increased Fndc5 and Pgc-1 $\alpha$ expressions in a dose-dependent manner (Fig. 1a and 1b). Similarly, increasing the cellular cAMP content by forskolin (a stimulator of adenylyl cyclase) [18] increased Fndc5 (Fig. 1c and 1d) and Pgc-1 $\alpha$

Fig. 1. cAMP increased Fndc5/Pgc-1 $\alpha$ expression in myotubes. (a-b) Myotubes were treated with various concentrations of Sp-cAMP for $24 \mathrm{~h}$. The transcription levels of Fndc5 (a) and Pgc-1 $\alpha$ (b) were measured by qRT-PCR ( $\mathrm{n}=3,{ }^{* *}: \mathrm{P}<0.01$ vs control, one-way ANOVA). (c) Fndc5 expression in $\mathrm{C} 2 \mathrm{C} 12$ myotubes after stimulated with forskolin at various concentrations for $24 \mathrm{~h}$ was measured by qRTPCT ( $n=3,{ }^{* *}$ : $\mathrm{P}<0.01$ vs control, one-way ANOVA). (d) Fndc5 expression in C2C12 myotubes after stimulated with $50 \mu \mathrm{M}$ forskolin for various time intervals was measured by qRTPCT $(\mathrm{n}=3$, **: $\mathrm{P}<0.01$ vs control, one-way ANOVA). (e) $P g c-1 \alpha$ expression in $\mathrm{C} 2 \mathrm{C} 12$ myotubes after stimulated with forskolin at various concentrations for $24 \mathrm{~h}$ was measured by qRT-PCT $(\mathrm{n}=3, *: \mathrm{P}<0.05, * *: \mathrm{P}<0.01$ vs control, one-way ANOVA). (f) $P g c-1 \alpha$ expression in $\mathrm{C} 2 \mathrm{C} 12$ myotubes after stimulated with $50 \mu \mathrm{M}$ forskolin for various time intervals was measured by qRT-PCT $\left(\mathrm{n}=3,{ }^{* *}\right.$ : $\mathrm{P}<0.01$ vs control, one-way ANOVA). (g) Fndc5 expression in C2C12 myotubes after isoproterenol $(10 \mu \mathrm{M})$ stimulation for 24 $\mathrm{h}$ was measured by qRT-PCT ( $\mathrm{n}=3,{ }^{* *}: \mathrm{P}<0.01$ vs control, one-way ANOVA).

\section{a}

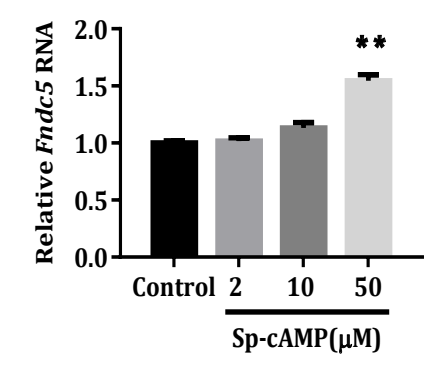

C

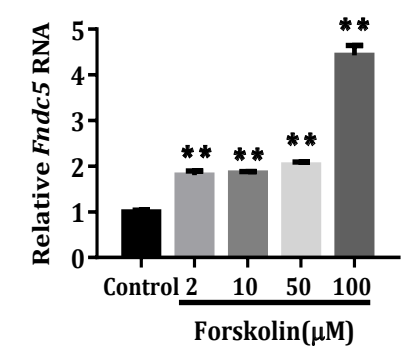

e

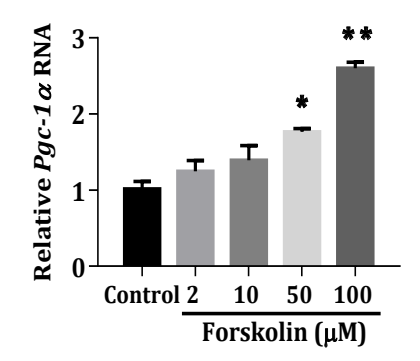

g

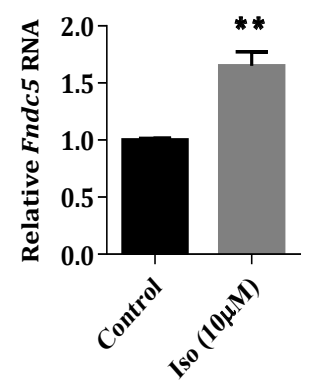

\section{b}

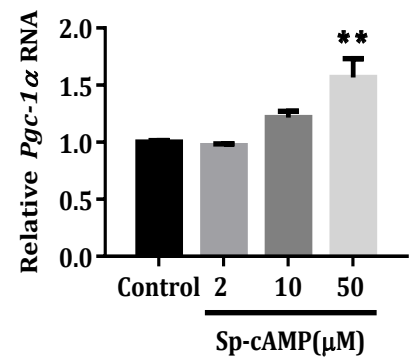

d

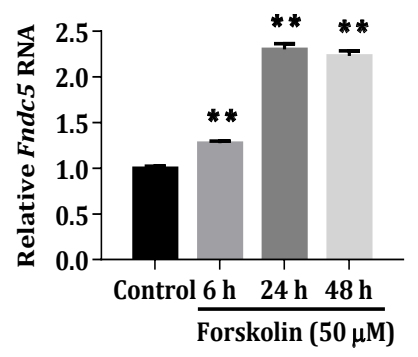

f

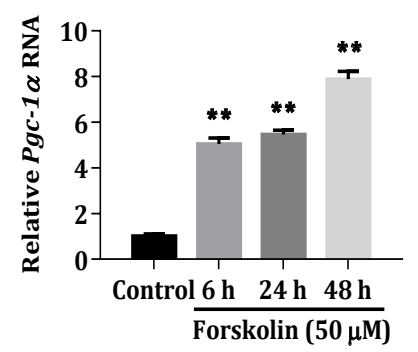


expressions (Fig. 1e and 1f). Isoproterenol is a non-selective $\beta$ adrenoreceptor agonist that also elevates the intracellular cAMP level $[19,20]$. Because $\beta$ adrenoreceptor activation is associated with exercise [21], isoproterenol is commonly used as a pharmacological agent to study the exercise-induced metabolic changes in various tissues [22, 23]. Interestingly, isoproterenol stimulation provoked a comparable induction of Fndc5 to that induced by 50 $\mu \mathrm{M}$ forskolin challenge (Fig. 1g). These data suggest that exercise-induced Fndc5 expression is possibly mediated via the cAMP signalling.

\section{CREB overexpression increased Fndc5 expression in myotubes}

cAMP response element-binding protein (CREB) is a cAMP-regulated transcription factor that controls energy homeostasis. In $\mathrm{C} 2 \mathrm{C} 12$ myotubes, forskolin or isoproterenol stimulation increased CREB phosphorylation (Fig. 2a). Suppressing, forskolin-induced Fndc5 expression was diminished when the CREB was inhibited by cyclosporine (Fig. 2b), suggesting CREB plays a role in regulating Fndc5 expression. When wild-type CREB or CREB S133A mutant was expressed in $\mathrm{C} 2 \mathrm{C} 12$ myotubes (Fig. 2c), augmented Fndc5 expression was only observed in wild-type CREB-transfected cells (Fig. 2d). We also found that overexpression of wild-type CREB, but not the CREB S133A mutant, in C2C12 myotubes upregulated Pgc-1 $\alpha$ expression (Fig. 2e), which aligned with the previous report that CREB was a transcriptional regulator of $P G C-1 \alpha$ expression [24].

\section{$P G C-1 \alpha$ increased Fndc5 expression through interacting with CREB in myotubes}

While over-expression of wild-type PGC- $1 \alpha$ increased Fndc5 expression, the presence of PGC-1 $\alpha$ dominant-negative (S570A) mutant reduced the levels of Fndc5 in C2C12 myotubes (Fig. 3a), confirming that PGC- $1 \alpha$ activity is critical for FNDC5 expression $[2,8]$. Interesting, co-expression of inactive CREB also abolished PGC-1 $\alpha$-induced Fndc5 expression (Fig. 3a),

Fig. 2. CREB

o ver expression increased Fndc5 and Pgc-1 $\alpha \quad$ expression in myotubes. C2C12 myotubes were stimulated with isoproterenol $(10 \mu \mathrm{M})$ or forskolin $(50 \mu \mathrm{M})$ for $30 \mathrm{~min}$ and the phosphorylation of CREB was examined by Western blotting. (b) Fndc5 expression in forskolin-stimulated C2C12 myotubes with or without cyclosporine A pre-treatment was detected by qRT-PCR $(\mathrm{n}=3, \quad *$ : $\mathrm{P}<0.05, \quad * *$ : $\mathrm{P}<0.01$, Student's t-test).

(c) Expressions of various CREB constructs and tubulin in $\mathrm{C} 2 \mathrm{C} 12$ myotubes were verified by immunoblotting. (d) Fndc5 expression in wild-type CREB or CREB S133A mutanttransfected C2C12 myotubes were examined by qRT-PCT ( $\mathrm{n}=3,{ }^{* *}$ : $\mathrm{P}<0.01$ vs control, one-was ANOVA). (e) Pgc-1 $\alpha$ expression in wild-type CREB or CREB S133A mutant-transfected C2C12 myotubes were examined by qRT-PCT $\left(\mathrm{n}=3,{ }^{* *}\right.$ : $\mathrm{P}<0.01$ vs control, one-was ANOVA).
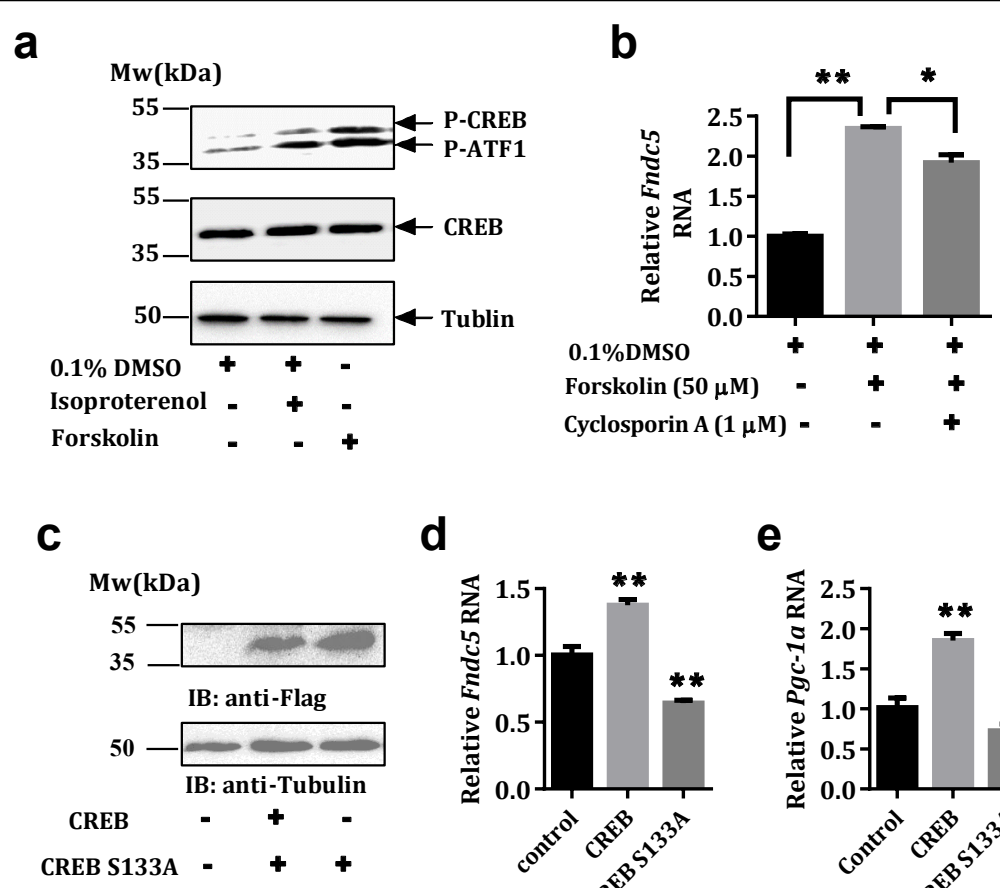

d

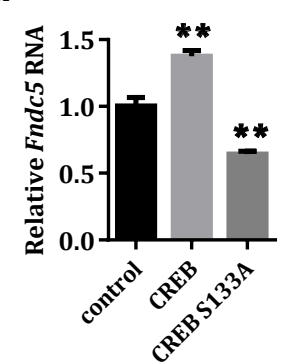

e

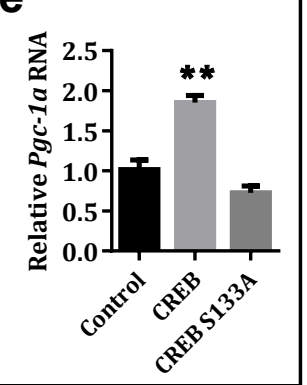
KARGER 
Fig. 3. PGC-1 $\alpha$ increased Fndc5 expression through interacting with CREB in myotubes. (a) Expression of Fndc5 in various PGC- $1 \alpha$ or CREB mutants-expressed C2C12 myotubes were determined by qRTPCR $\quad(n=3, \quad * *: \quad \mathrm{P}<0.01$ vs control, one-way ANOVA). (b) Coimmunoprecipitation of overexpressed PGC$1 \alpha$ and CREB in HEK 293 cells. Lysates from the transfected HEK 293 cells were immunoprecipitated with anti-Flag antibody. The pulled-down proteins were analysed by immunoblotting. Expression of CREB and

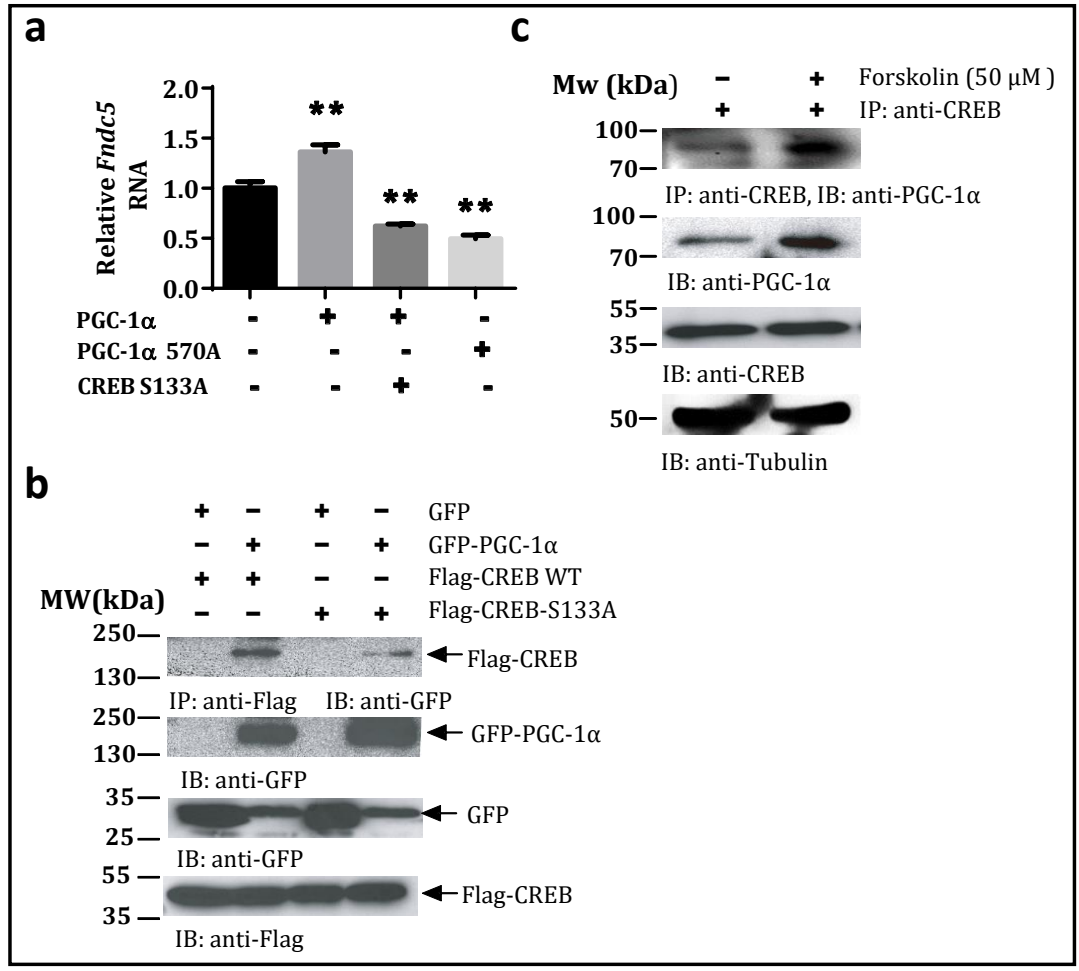
tubulin were also verified by Western blotting. Expression of GFP-PGC- $1 \alpha$, Flag-CREBs, and tubulin were also verified by immunoblotting. (c) Co-immunoprecipitation of endogenous PGC-1 $\alpha$ and CREB in C2C12 myotubes treated with forskolin $(50 \mu \mathrm{M}, 24 \mathrm{~h})$. C2C12 lysates were immunoprecipitated with anti-CREB antibody and the pulled-down proteins were analysed by SDS-PAG using anti-PGC-1 $\alpha$ antibody. Expression of PGC-1 $\alpha$, CREB, and tubulin were also verified by Western blotting.

suggesting that PGC-1 $\alpha$ may interact with CREB to control Fndc5 expression. Agreeing with this hypothesis, immunoprecipitation showed that PGC-1 $\alpha$ complexed with CREB, and the presence of CREB S133 mutant downregulated their interaction (Fig. 3b). The endogenous CREB/PGC- $1 \alpha$ interaction could be readily detected in C2C12 myotubes, which was increased after forskolin stimulation (Fig. 3c).

\section{Irisin increased mitochondrial respiration in myotubes}

To investigate the metabolic functions of irisin, we monitored the oxidative and glycolytic metabolism in C2C12 myotubes. Treatment with various concentrations of irisin $(10,50$, $100 \mathrm{ng} / \mathrm{ml}$ ) for $24 \mathrm{~h}$ increased the oxygen consumption rate (OCR) (Fig. 4a), ATP production (Fig. 4b), basal respiration (Fig. 4c), and maximal respiration (Fig. 4d). The ATP-coupling efficiency was not altered after irisin stimulation (Fig. 4e), suggesting the increased ATP production was not caused by enhanced enzymatic activities in oxidative phosphorylation but an augmented mitochondrial number. Indeed, treatment with irisin significantly provoked the expression of Pgc-1 $\alpha$, Tfam, and Nrf1 expressions in C2C12 myotubes (Fig. 4f), which are the key transcriptional regulators of mitochondrial biogenesis [25]. On the other hand, irisin stimulation lowered basal ECAR (Fig. $4 \mathrm{~g}$ and $4 \mathrm{~h}$ ). Therefore, the OCR/ECAR ratio in C2C12 myotubes was significantly augmented after irisin stimulation (Fig. 4i), which represented a glycolysis-to-oxidative phosphorylation shift for cellular ATP generation [26].

\section{Irisin increased myotube fatty acid oxidation}

Exercise increases free fatty acid (FFA) uptake and oxidation in muscle [27]. To test whether exercise-induced irisin production is responsible for fatty acid metabolism in skeletal muscle, we evaluated the effect of irisin on FFA oxidation. C2C12 myotubes were 
Fig. 4. Irisin increased m i t o c h o n d ria l respiration but decreases extracellular acidification in $\mathrm{C} 2 \mathrm{C} 12$ myotubes. (a-e) After treated with irisin for $24 \mathrm{~h}$, the kinetic profile of mitochondrial respiration (a), ATP production (b), basal respiration (c), maximal respiration, (d) maximal respiration, and (e) coupling efficiency of $\mathrm{C} 2 \mathrm{C} 12$ myotubes were determined by the Extracellular Flux analyser (n = 4, *: $\mathrm{P}<0.05, \quad * *$ : $\mathrm{P}<0.001$, one-way ANOVA). (f) Expression of Pgc$1 \alpha$, Tfam and Nrf1 in irisin-stimulated (24 h) C2C12 myotubes were measured by qRTPCR (n=3, *: $\mathrm{P}<0.05$, **: $\quad \mathrm{P}<0.01$, Student's t-test). (g-h) After treated with irisin for $24 \mathrm{~h}$, the kinetic profile (g) and basal ECAR (h) of $\mathrm{C} 2 \mathrm{C} 12$ myotubes were detected by the Extracellular Flux analyser $(\mathrm{n}=4$, *: $\quad \mathrm{P}<0.05$, one-way ANOVA). (i) OCR to ECAR ratio of $\mathrm{C} 2 \mathrm{C} 12$ myotubes after irisin treatment $(24 \mathrm{~h})$ as recorded by the Extracellular Flux Analyser $(\mathrm{n}=4, *$ : $\mathrm{P}<0.05, * *$ : $\mathrm{P}<0.001$, one-way ANOVA).

stimulated by $100 \mathrm{ng} / \mathrm{ml}$ of irisin for $24 \mathrm{~h}$, and FFA oxidation was measured using OCR as an indicator [28]. We found that irisin increased cellular oxygen consumption when palmitic acid was supplied as the sole energy source (Fig. 5a). The ATP production (Fig. 5b), basal respiration (Fig. 5c), and maximal respiration (Fig. 5d) of myotubes were also elevated.

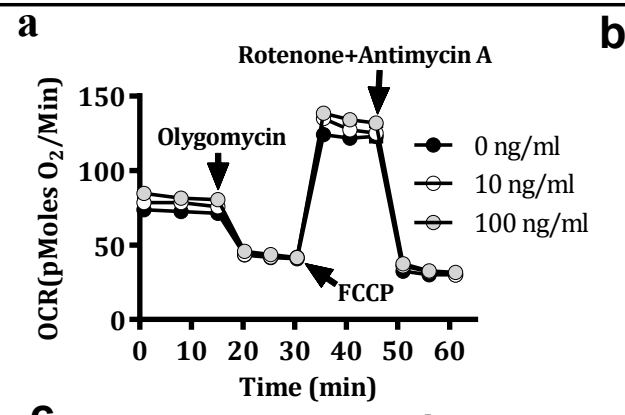

b
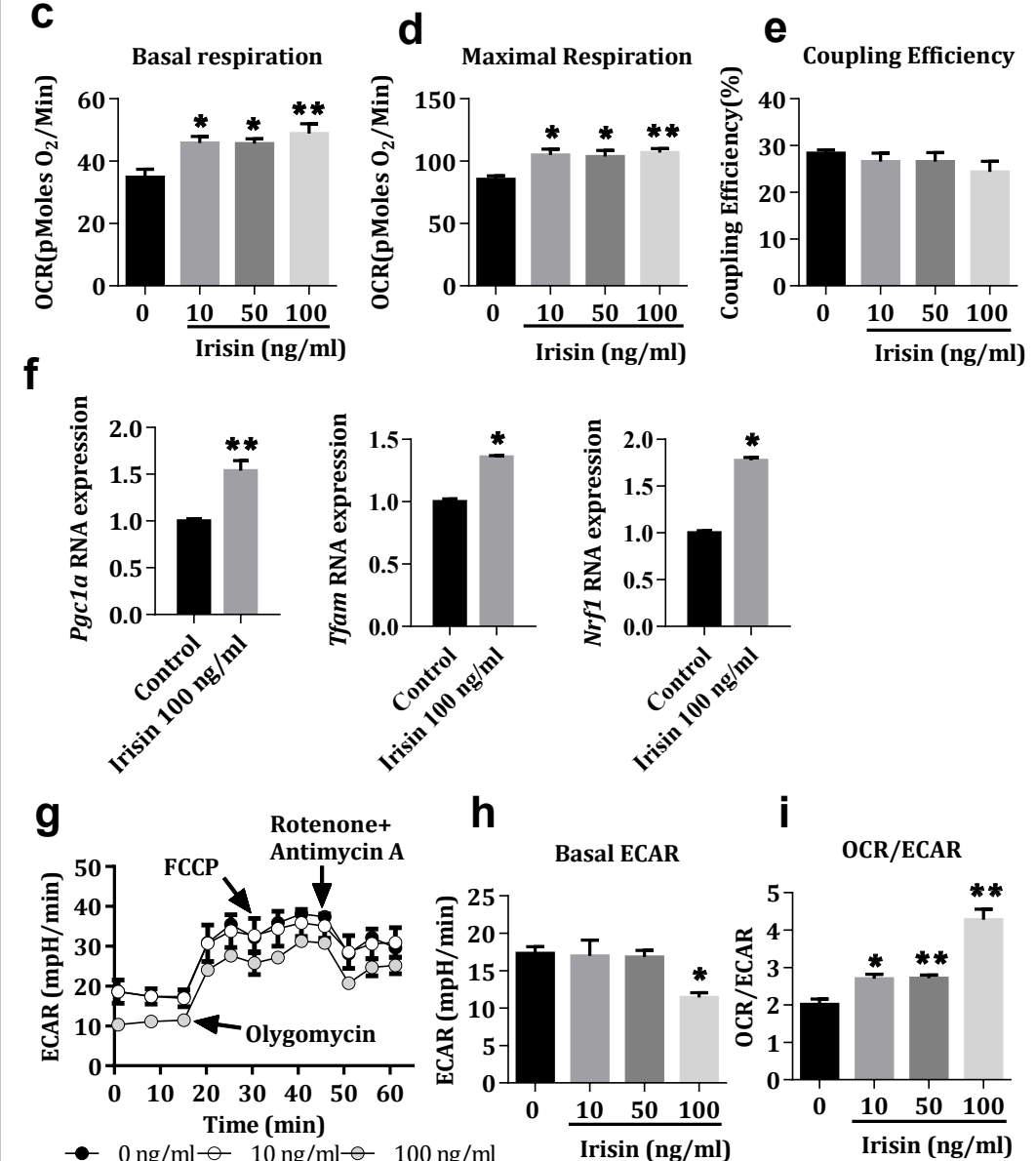

h
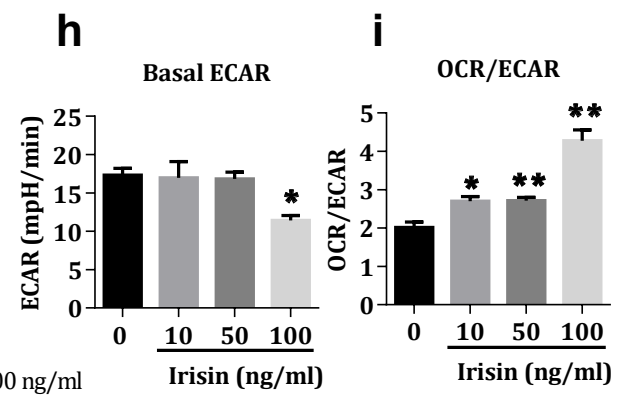


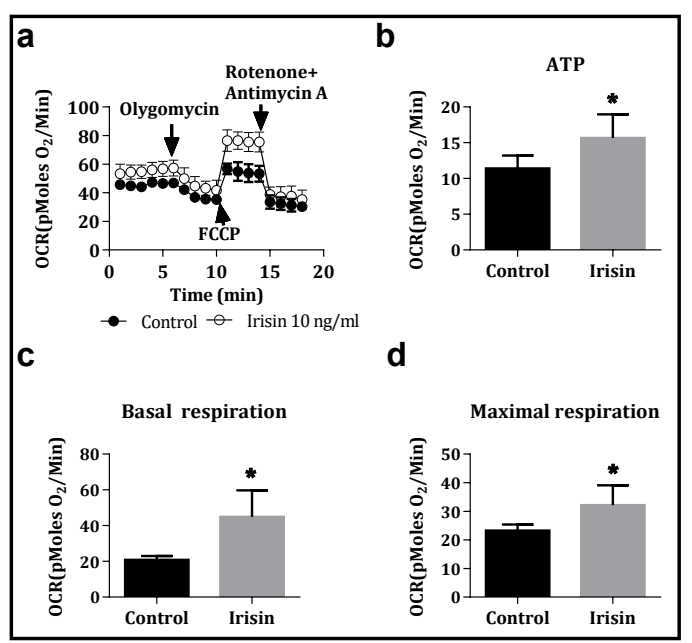

Fig. 5. Irisin increased fatty acid oxidation in C2C12 myotubes. After stimulated with irisin for 24 $\mathrm{h}$, the kinetic profile of mitochondrial respiration (a), ATP production (b), basal respiration (c), and maximal respiration (d) in the presence of palmitic acid $(200 \mu \mathrm{M})$ were recorded by the Extracellular Flux Analyser ( $\mathrm{n}=4$, *: $^{\mathrm{P}<0.05}$, Student's t-test).

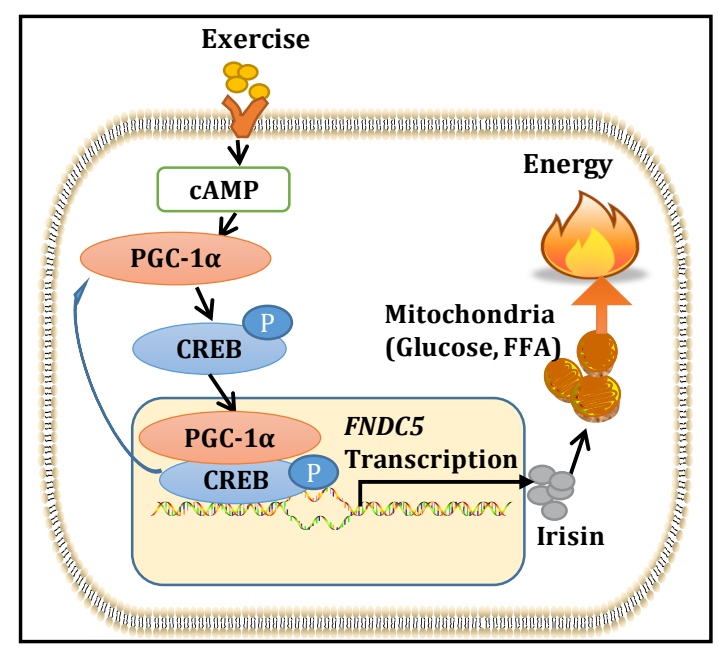

Fig. 6. Proposed model of exercise-induced FNDC5 expression. Exercise increases the intracellular content of cAMP, which triggers the CREB/PGC- $1 \alpha$ interaction to induce the transcription FNDC5. The newly synthesized irisin will serve as an autocrine/ paracrine to enhance mitochondrial respiration and FFA oxidation in skeletal muscle.

\section{Discussion}

Skeletal muscle is not only responsible for locomotion but also acts as an endocrine organ [29]. In response to muscular contractions or different metabolic demands, it secretes myokines to communicate with other tissues $[29,30]$. Irisin is one of the most prominent metabolic myokines that is generated from contracting muscle during exercise [31]. Previous studies have demonstrated that irisin stimulation increased the metabolic rate, mitochondrial content $[6,32]$, and FFA oxidation in myocytes $[3,7]$. Moreover, a positive correlation of mortality risk in acute heart failure and serum irisin was also observed, suggesting irisin can be used as a predictive biomarker for cardiovascular diseases [33]. In this study, we demonstrated that exercise may enhance cAMP to stimulate muscular Pgc-1 $\alpha$ expression, which interacts with the transcription factor CREB to induce Fndc5 expression. Consequently, irisin may serve as an autocrine/paracrine to increase mitochondrial respiration and shift the metabolic preference from glucose to FFA for ATP production in muscle cells (Fig. 6).

The signalling pathways that control FNDC5 expression have not been fully elucidated with contradictory results from different in vitro studies [10,11]. For instance, it was reported that exercise-mimicking treatment failed to increase Fndc5 expression in myotubes [34]. Adrenaline stimulation also showed no significant effects on Fndc5 expression in cultured myotubes [17]. These conflicting results might be attributed to the difference in experimental protocol or choice of anti-irisin antibodies [10]. Nevertheless, our study demonstrated that cAMP treatment increased both Fndc5 and Pgc-1 $\alpha$ expressions in myotubes, probably because of longer stimulation $(24 \mathrm{~h})$ than in other in vitro models $(1 \mathrm{~h})[6,32]$.

Skeletal muscle-specific Pgc-1 $\alpha$ knockout animals show reduced endurance capacity as well as other signs of motility defects and metabolic dysfunctions $[35,36]$. On the other hand, over expressing Pgc-1 $\alpha$ in muscle increased Fndc5 expression and irisin release, eventually causing higher UCP1-dependent thermogenesis and energy expenditure [2]. In our study, we found that Pgc-1 $\alpha$ over expression induced Fndc5 expression in myotubes. We also proved that CREB promoted both Fndc5 and Pgc-1 $\alpha$ expression. More importantly, CREB inactivation suppressed PGC-1 $\alpha$-induced Fndc5 transcription. The results suggest that 
intact CREB is essential for PGC- $1 \alpha$ to control Fndc5 transcription. Previous studies have reported that CREB controls the transcription of $P G C-1 \alpha$ through binding to its promoter [37]. Our results further suggest that PGC- $1 \alpha$ may physically interact with the transcription factor CREB to stimulate Fndc5 expression.

\section{Conclusion}

Our study demonstrates that the expression of Fndc5 in skeletal muscle is mediated by the cAMP-induced PGC-1 $\alpha /$ CREB interaction. Our functional studies also indicated that irisin shapes the metabolic phenotype of myotubes by increasing fatty acid oxidation but reducing glycolysis.

\section{Acknowledgements}

This work was supported by grants from the Hong Kong Government Research Grant Council (ECS 27100816) to C.B. Chan, and the CAMS Initiative for Innovative Medicine (CAMS-I2M 2016-I2M-3-007, 2017-I2M-1-010) and National Natural Science Foundation of China $(81470159,81770847)$ to X. Yang.

\section{Disclosure Statement}

The authors declare no conflicts of interest.

\section{References}

1 Huh JY, Panagiotou G, Mougios V, Brinkoetter M, Vamvini MT, Schneider BE, Mantzoros CS: FNDC5 and irisin in humans: I. Predictors of circulating concentrations in serum and plasma and II. mRNA expression and circulating concentrations in response to weight loss and exercise. Metabolism 2012;61:1725-1738.

- Boström P, Wu J, Jedrychowski MP, Korde A, Ye L, Lo JC, Rasbach KA, Boström EA, Choi JH, Long JZ, Kajimura S, Zingaretti MC, Vind BF, Tu H, Cinti S, Højlund K, Gygi SP, Spiegelman BM: A PGC1- $\alpha$-dependent myokine that drives brown-fat-like development of white fat and thermogenesis. Nature 2012;481:463-468.

- Perakakis N, Triantafyllou GA, Fernandez-Real JM, Huh JY, Park KH, Seufert J, Mantzoros CS: Physiology and role of irisin in glucose homeostasis. Nat Rev Endocrinol 2017;13:324-337.

4 Hou N, Du G, Han F, Zhang J, Jiao X, Sun X: Irisin Regulates Heme Oxygenase-1/Adiponectin Axis in Perivascular Adipose Tissue and Improves Endothelial Dysfunction in Diet-Induced Obese Mice. Cell Physiol Biochem 2017;42:603-614.

-5 Huh JY, Mougios V, Kabasakalis A, Fatouros I, Siopi A, Douroudos II, Filippaios A, Panagiotou G, Park KH, Mantzoros CS: Exercise-induced irisin secretion is independent of age or fitness level and increased irisin may directly modulate muscle metabolism through AMPK activation. J Clin Endocrinol Metab 2014;99:E2154-2161.

-6 Vaughan RA, Gannon NP, Barberena MA, Garcia-Smith R, Bisoffi M, Mermier CM, Conn CA, Trujillo KA: Characterization of the metabolic effects of irisin on skeletal muscle in vitro. Diabetes Obes Metab 2014;16:711-718.

7 Xin C, Liu J, Zhang J, Zhu D, Wang H, Xiong L, AUID- Oho, Lee Y, Ye J, Lian K, Xu C, Zhang L, Wang Q Liu Y, Tao L: Irisin improves fatty acid oxidation and glucose utilization in type 2 diabetes by regulating the AMPK signaling pathway. Int J Obes (Lond) 2016;40:443-451.

$>8$ Shan T, Liang X, Bi P, Kuang S: Myostatin knockout drives browning of white adipose tissue through activating the AMPK-PGC1 $\alpha$-Fndc5 pathway in muscle. FASEB J 2013;27:1981-1989.

-9 Pilegaard H, Saltin B, Neufer PD: Exercise induces transient transcriptional activation of the PGC-1alpha gene in human skeletal muscle. J Physiol 2003;546:851-858. 


\section{Cellular Physiology Cell Physiol Biochem 2018;50:1574-1584 \begin{tabular}{l|l|l} 
and Biochemistry Published online: 26 October 2018 & $\begin{array}{l}\text { ○ 2018 The Author(s). Published by S. Karger AG, Basel } \\
\text { www.karger.com/cpb }\end{array}$ \\
\hline
\end{tabular}}

Yang et al.: CREB and PGC-1 $\alpha$ Control FNDC5 Expression

10 Zhang J, Valverde P, Zhu X, Murray D, Wu Y, Yu L, Jiang H, Dard MM, Huang J, Xu Z, Tu Q Chen J: Exerciseinduced irisin in bone and systemic irisin administration reveal new regulatory mechanisms of bone metabolism. Bone Res 2017;5:16056.

11 Dinas PC, Lahart IM, Timmons JA, Svensson PA, Koutedakis Y, Flouris AD, Metsios GS: Effects of physical activity on the link between PGC-1a and FNDC5 in muscle, circulating Iotarisin and UCP1 of white adipocytes in humans: A systematic review. F1000Res 2017;6:286.

12 Burattini S, Ferri P, Battistelli M, Curci R, Luchetti F, Falcieri E: C2C12 murine myoblasts as a model of skeletal muscle development: morpho-functional characterization. Eur J Histochem 2004;48:223-233.

$\checkmark 13$ Radonić A, Thulke S, Mackay IM, Landt O, Siegert W, Nitsche A: Guideline to reference gene selection for quantitative real-time PCR. Biochem Biophys Res Commun 2004;313:856-862.

14 Lawrence JC Jr, Salsgiver WJ: Evidence that levels of malate dehydrogenase and fumarase are increased by cAMP in rat myotubes. Am J Physiol 1984;247:C33-38.

15 Costford SR, Bajpeyi S, Pasarica M, Albarado DC, Thomas SC, Xie H, Church TS, Jubrias SA, Conley KE, Smith SR: Skeletal muscle NAMPT is induced by exercise in humans. Am J Physiol Endocrinol Metab 2010;298:E117-126.

16 Sparks LM, Moro C, Ukropcova B, Bajpeyi S, Civitarese AE, Hulver MW, Thoresen GH, Rustan AC, Smith SR: Remodeling lipid metabolism and improving insulin responsiveness in human primary myotubes. PLoS One 2011;6:e21068.

17 Sanchez J, Nozhenko Y, Palou A, Rodriguez AM: Free fatty acid effects on myokine production in combination with exercise mimetics. Mol Nutr Food Res 2013;57:1456-1467.

18 Alasbahi RH, Melzig MF: Forskolin and derivatives as tools for studying the role of cAMP. Pharmazie 2012;67:5-13.

-19 Lerman BB, Belardinelli L, West GA, Berne RM, DiMarco JP: Adenosine-sensitive ventricular tachycardia: evidence suggesting cyclic AMP-mediated triggered activity. Circulation 1986;74:270-280.

-20 Piot C, LeMaire SA, Albat B, Seguin J, Nargeot J, Richard S: High frequency-induced upregulation of human cardiac calcium currents. Circulation 1996;93:120-128.

-21 Thompson D, Karpe F, Lafontan M, Frayn K: Physical activity and exercise in the regulation of human adipose tissue physiology. Physiol Rev 2012;92:157-191.

-22 Richards JC, Johnson TK, Kuzma JN, Lonac MC, Schweder MM, Voyles WF, Bell C: Short-term sprint interval training increases insulin sensitivity in healthy adults but does not affect the thermogenic response to beta-adrenergic stimulation. J Physiol 2010;588:2961-2972.

23 Lessard SJ, Rivas DA, Chen ZP, van Denderen BJ, Watt MJ, Koch LG, Britton SL, Kemp BE, Hawley JA: Impaired skeletal muscle beta-adrenergic activation and lipolysis are associated with whole-body insulin resistance in rats bred for low intrinsic exercise capacity. Endocrinology 2009;150:4883-4891.

24 Xie K, Zhu M, Xiang P, Chen X, Kasimumali A, Lu R, Wang Q Mou S, Ni Z, Gu L, Pang H: PKA/CREB signaling prevents adriamycin-induced podocyte apoptosis via upregulation of mitochondrial respiratory chain complexes. Mol Cell Biol 2017;38:pii:e00181-00117.

-25 Scarpulla RC, Vega RB, Kelly DP: Transcriptional integration of mitochondrial biogenesis. Trends Endocrinol Metab 2012;23:459-466.

-26 Yizhak K, Le DSE, Rogkoti VM, Baenke F, de Boer VC, Frezza C, Schulze A, van de Water B, Ruppin E: A computational study of the Warburg effect identifies metabolic targets inhibiting cancer migration. Mol Syst Biol 2014;10:744.

27 Jensen MD: Fatty acid oxidation in human skeletal muscle. J Clin Invest 2002;110:1607-1609.

-28 MCL T, Herlea-Pana O, Brobst D, Yang X, Wood J, Hu X, Liu Z, Lee CW, Zaw AM, BKC C, Ye K, Chan CB: Tumor Necrosis Factor-alpha Promotes Phosphoinositide 3-Kinase Enhancer A and AMP-Activated Protein Kinase Interaction to Suppress Lipid Oxidation in Skeletal Muscle. Diabetes 2017;66:1858-1870.

29 Schnyder S, Handschin C: Skeletal muscle as an endocrine organ: PGC-1 $\alpha$, myokines and exercise. Bone 2015;80:115-125.

-30 Pedersen BK, Akerström TC, Nielsen AR, Fischer CP: Role of myokines in exercise and metabolism. J Appl Physiol (1985) 2007;103:1093-1098.

31 Chen N, AUID- Oho, Li Q, Liu J, Jia S: Irisin, an exercise-induced myokine as a metabolic regulator: an updated narrative review. Diabetes Metab Res Rev 2016;32:51-59.

-32 Vaughan RA, Gannon NP, Mermier CM, Conn CA: Irisin, a unique non-inflammatory myokine in stimulating skeletal muscle metabolism. J Physiol Biochem 2015;71:679-689. 


\section{Cellular Physiology Cell Physiol Biochem 2018;50:1574-1584

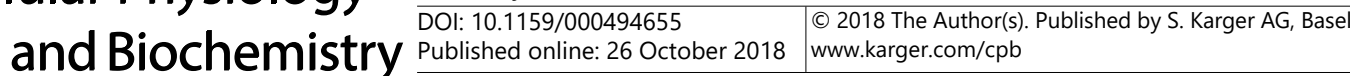

Yang et al.: CREB and PGC- $1 \alpha$ Control FNDC5 Expression

33 Shen S, Gao R, Bei Y, Li J, Zhang H, Zhou Y, Yao W, Xu D, Zhou F, Jin M, Wei S, Wang K, Xu X, Li Y, Xiao J, Li X: Serum Irisin Predicts Mortality Risk in Acute Heart Failure Patients. Cell Physiol Biochem 2017;42:615622.

-34 Kurdiova T, Balaz M, Mayer A, Maderova D, Belan V, Wolfrum C, Ukropec J, Ukropcova B: Exercisemimicking treatment fails to increase Fndc5 mRNA \& irisin secretion in primary human myotubes. Peptides 2014;56:1-7.

-35 Handschin C, Chin S, Li P, Liu F, Maratos-Flier E, Lebrasseur NK, Yan Z, Spiegelman BM: Skeletal muscle fiber-type switching, exercise intolerance, and myopathy in PGC-1 alpha muscle-specific knock-out animals. J Biol Chem 2007;282:30014-30021.

-36 Handschin C, Choi CS, Chin S, Kim S, Kawamori D, Kurpad AJ, Neubauer N, Hu J, Mootha VK, Kim YB, Kulkarni RN, Shulman GI, Spiegelman BM: Abnormal glucose homeostasis in skeletal muscle-specific PGC1alpha knockout mice reveals skeletal muscle-pancreatic beta cell crosstalk. J Clin Invest 2007;117:34633474.

37 Handschin C, Rhee J, Lin J, Tarr PT, Spiegelman BM: An autoregulatory loop controls peroxisome proliferator-activated receptor gamma coactivator 1alpha expression in muscle. Proc Natl Acad Sci U S A 2003;100:7111-7116. 